\title{
Dynamic Poverty Measures
}

\author{
Eugene Kouassi ${ }^{1}$, Pierre Mendy ${ }^{2}$, Diaraf Seck ${ }^{2}$, Kern O. Kymn ${ }^{3 *}$ \\ ${ }^{1}$ Resource Economics, West Virginia University, Morgantown, USA \\ ${ }^{2}$ Faculty of Economics, Cheikh Anta Diop University, Dakar, Senegal \\ ${ }^{3}$ Division of Finance and Economics, West Virginia University, Morgantown, USA \\ E-mail: kern.kymn@mail.wvu.edu \\ Received June 9, 2011; revised August 7, 2011; accepted August 15, 2011
}

\begin{abstract}
In this paper we propose methods for detecting the number of pores based on dynamic optimization techniques. An illustration is provided and the results are discussed based on Government's objectives and control variables.
\end{abstract}

Keywords: Optimization Techniques, Poverty Measures

\section{Introduction}

How poverty is measured is a central topic in economic and policy analyses. However, recently, it has clearly appeared that it is not only the determination of particular poverty levels at particular instants (based on several indices available in the literature) that matter the most. The paths of poverty levels over time are also critical and crucial indicators in assessing the efficiency of poverty measures (Ciarlet, 2006 [1]).

This paper adds to the literature on this topic by providing methods for measuring poverty in dynamic environment (Dia and Popescu, 1996 [2]). The proposed approach answers the following question: How important are dynamic optimization techniques for poverty measure and analysis?

The remainder of the paper is organized as follows: In Section 2, we address the problem of poverty measures in a dynamic context based on optimal control. An illustration is provided and discussed in Section 3. Finally, some concluding remarks appear in Section 4.

\section{Dynamic Poverty Measures}

In the context of dynamic optimization, time does matter and the paths of poverty indexes are important.

\subsection{The Problem in Discrete Time}

Consider $t_{0}$ an initial instant. We assume that a static model has been used to determine the number of pores in a given population based on a given poverty line. Let $t$ be the time index, $Y_{t} \in \mathbb{R}_{+}^{* Q}$, the vector of revenues of the $Q$ pores who have been identified at time $t$. Let $u_{t} \in \mathbb{R}^{p}$, be the vector of the $p$ control variables which represent the set of commands. The objective is to measure the performance of the system. To this end, we consider an objective function to optimize subject to some constraints. The above problem can be formalized as follows (Rustagi, 1997 [3])

$$
\begin{aligned}
& \text { Opt } J, \quad J:=\sum_{t=t_{0}}^{T-1} k\left(Y_{t}, a_{t}, u_{t}, t\right) \\
& Y_{t+1}=g_{t}\left(Y_{t}, u_{t}\right), t=t_{0}, t_{1}, \cdots, T-1
\end{aligned}
$$

where the second equality represents the constraints on the state vector which is the vector of revenues $Y_{t}, a_{t}$ the objective at each period of time $t$ and $u_{t}$ the control variables. The problem now is to choose the best control vector $u_{t}$, at each period of time, according to available resources, such that the above system is satisfied (Rustagi, 1997 [3] and Mart, 1997 [4]). In this type of problem, it is the final stage which is the most important since the objective is to reduce or eliminate poverty. This of course depends on intermediary objectives. More specifically, the dynamic optimization problem can be set up as a minimization problem of $J$ (Troutman, 1980 [5]),

$$
\begin{aligned}
\min J, J & :=\sum_{t=t_{0}}^{T-1} k\left(Y_{t}, a_{t}, u_{t}, t\right) \\
& =\sum_{t=t_{0}}^{T-1}\left(Y_{t}-a_{t}\right)\left(Y_{t}-a_{t}\right)^{t}+u_{t} u_{t}^{t}
\end{aligned}
$$

How to justify the choice of the function $k\left(Y_{t}, a_{t}\right.$, $\left.u_{t}, t\right)$ ? In the above problem, it is the final revenue 
which is the most important, i.e., $Y_{T-1}$. In fact, the objective is to get the individuals in the vector of revenues $Y_{T-1}$ out of poverty. Therefore, intermediary objectives to be reached and any set of decisions at time $T-1$ should be such that, $Y_{i}^{t} \geq Z, \forall i$, where $Z$ is the poverty line. From a mathematical point of view, at $T-1$, the Euclidian norm of $a_{T-1}$, i.e., $\left\|a_{T-1}\right\|$ must be at least of the same order as $N Z^{2}$, the poverty line of the population under consideration. To this end, it suffices to prove that,

$$
\begin{array}{ll} 
& \left\|\sum_{i=1}^{Q}\left(a_{i}^{T-1}\right)^{2}-N Z^{2}\right\| \geq 1 \\
\text { or } & \left\|\sum_{i=1}^{Q}\left(a_{i}^{T-1}\right)^{2}-N Z^{2}\right\|=o(1)
\end{array}
$$

where $o$ is the Landau notation.

Equation (4) has an immediate solution

$$
\begin{aligned}
& a_{i}^{t} \geq\left(\frac{t}{T-1}\right) \sqrt{\frac{1+N Z^{2}}{Q}}, \\
& i \in\{1, \cdots, Q\} ; t \in\left\{t_{0}, \cdots, T-1\right\}
\end{aligned}
$$

In addition, another constraint is that when $r \rightarrow N Z^{2}$, the level of richness of the individual $i, r$, must be in adequacy with the poverty line if not higher i.e., $Y_{i}$ must be higher than the poverty line. Therefore the constraint on the final objectives must be such that,

$$
\left\|\sum_{i=1}^{Q}\left(a_{i}^{T-1}\right)^{2}-N Z^{2}\right\| \geq 1
$$

Regarding the system to control, $Y_{t+1}=g_{t}\left(Y_{t}, u_{t}\right)$, $t=t_{0}, t_{1}, \cdots, T-1$ for simplicity, we consider a linear system. Therefore,

$$
Y_{t+1}=A_{t} Y_{t}+B_{t} u_{t}, t=t_{0}, t_{1}, \cdots, T-1
$$

where $A_{t}$ is a square matrix of order $Q$, i.e., $A_{t}=\left(a_{i j}^{t}\right)_{\substack{1 \leq i \leq Q \\ 1 \leq j \leq Q}}, B_{t}$ is a matrix with $Q$ rows and $p$ columns, i.e., $B_{t}=\left(b_{i j}^{t}\right)_{\substack{1 \leq i \leq Q \\ 1 \leq j \leq p}}$. These matrices can be identified using economic theory. Summarizing the discrete time problem we have (Troutman, 1980 [5]),

$$
\left\{\begin{array}{l}
\min J, J:=\sum_{t=t_{0}}^{T-1}\left(Y_{t}-a_{t}\right)\left(Y_{t}-a_{t}\right)^{t}+u_{t} u_{t}^{t} \\
\text { s.t. } \\
Y_{t+1}=A_{t} Y_{t}+B_{t} u_{t}, t=t_{0}, t_{1}, t_{2}, \cdots, T-1 \\
a_{i}^{t} \geq\left(\frac{t}{T-1}\right) \sqrt{\left(\frac{1+N Z^{2}}{Q}\right)} \\
\left\|\sum_{i=1}^{Q}\left(a_{i}^{T-1}\right)^{2}-N Z^{2}\right\| \geq 1 \\
i \in\{1, \cdots, Q\}, t \in\left\{t_{0}, \cdots, T-1\right\}
\end{array}\right.
$$

\subsection{The Problem in Continuous Time}

Based on the above developments, by analogy and with some minor modifications, in continuous time, the dynamic problem can be formalized as follows,

$$
\left\{\begin{array}{l}
\min J: J=\int_{t_{0}}^{T}\left(\|Y(t)-a(t)\|^{2}+\|u(t)\|^{2}\right) \mathrm{d} t \\
\text { s.t. } \\
\frac{\mathrm{d} Y(t)}{\mathrm{d} t}=A(t) Y(t)+B(t) u(t) \\
a(t) \geq \frac{t}{T}\left(\frac{1+N Z^{2}}{Q}\right) ; t \in\left[t_{0}, T\right] \\
u:\left[t_{0}, T\right] \rightarrow \mathbb{R}^{p} \text { a vector of continuous } \\
\text { step functions. } \\
\text { min } J=-\max (-J)
\end{array}\right.
$$

Set $G=-J$ and

$$
\begin{aligned}
& f_{0}\left(t_{0}, Y(t), u(t), a(t)\right) \\
& =-\|Y(t)-a(t)\|^{2}-\|u(t)\|^{2}
\end{aligned}
$$

\subsection{Solution}

To conserve space, the solution given here is related only to the continuous case. Deduction of the discrete time solution is then straightforward.

Let $V_{0}$ be the maximal value of the objective function. It is easy to verify that,

$$
\begin{aligned}
& V_{0}(t, Y)= \max \int_{t_{0}}^{T} f_{0}\left(s_{0}, Y(s), u(s), a(s)\right) \mathrm{d} s \\
&+V_{0}(t+\Delta, Y+\Delta) \\
& \text { given } u:[t, t+\Delta] \rightarrow \mathbb{R}^{p}, \Delta \geq 0 \\
& V(t, Y)=-V_{0}(t, Y) \\
&= \min \int_{t_{0}}^{T}-f_{0}\left(s_{0}, Y(s), u(s), a(s)\right) \mathrm{d} s \\
&-V(t+\Delta, Y+\Delta) \\
& \text { given } u:[t, t+\Delta] \rightarrow \mathbb{R}^{p}, \Delta \geq 0 \\
& \frac{\mathrm{d} Y(s)}{\mathrm{d} t}=A(s) Y(s)+B(s) u(s), \quad Y(s)=Y
\end{aligned}
$$

Let us assume that $V$ is differentiable in $t$ and $Y$. Then,

$$
\begin{aligned}
V(t, Y)=\min \{( & \left(\|Y(t)-a(t)\|^{2}+\|u(t)\|^{2}\right) \Delta \\
& +\Delta \frac{\partial V}{\partial Y}(A(t) Y(t)+B(t) u(t)) \\
& \left.+\frac{\partial V}{\partial t}(t, Y) \Delta+o(\Delta), u \in \mathbb{R}^{p}\right\}
\end{aligned}
$$


with $\Delta>0$ and where $\frac{\partial V}{\partial Y}=\nabla_{Y}$ and where $\nabla$ is the gradient operator. Dividing by $\Delta$ and letting $\Delta \rightarrow 0$, we get the Hamilton-Jacobi-Bellman partial derivative equation of the form,

$$
\frac{\partial V}{\partial t}+\min \left\{\begin{array}{l}
\left(\|Y(t)-a(t)\|^{2}+\|u(t)\|^{2}\right) \\
+\frac{\partial V}{\partial Y}(A(t) Y(t)+B(t) u(t))
\end{array}\right\}=0
$$

given $u \in \mathbb{R}^{p}$

We then have the following theorem:

\section{Theorem 1}

Assuming there exists a differentiable function $V$ : $\left[t_{0}, T\right] \times \mathbb{R}^{Q} \rightarrow \mathbb{R}$ which satisfies (12).

Assuming that: $\exists \Psi:\left[t_{0}, T\right] \times \mathbb{R}^{Q} \rightarrow \mathbb{R}^{p}$ with $\Psi$ a continuous function in $t$ and Lipschitz in $Y$ and satisfying,

$$
\begin{aligned}
& \|Y(t)-a(t)\|^{2}+\|\Psi(t, Y)\|^{2} \\
& +\frac{\partial V}{\partial Y}(A(t) Y(t)+B(t) \Psi(t, Y)) \\
= & \min \left\{\left(\|Y(t)-a(t)\|^{2}+\|u(t)\|^{2}\right)\right. \\
& \left.+\frac{\partial V}{\partial Y}(A(t) Y(t)+B(t) u(t))\right\}
\end{aligned}
$$

$$
\text { given } u \in \mathbb{R}^{p}
$$

Then, $\Psi$ is a control optimal feedback for problem (13), i.e., $V$ is the minimum of $J$.

Proof: (See the Appendix).

In the continuous case, by analogy to the discrete case, the general model proposed here is,

$$
\left\{\begin{array}{l}
\text { Opt : } \quad J=\int_{t_{0}}^{T} k(Y(t), a(t), u(t), t) \mathrm{d} t \\
\text { s.t. } \\
\frac{\mathrm{d} Y(t)}{\mathrm{d} t}=g(Y(t), u(t), t), t \in\left[t_{0}, T\right]
\end{array}\right.
$$

The specific model proposed is,

$$
\left\{\begin{array}{l}
\min \mathrm{J}: J=\int_{t_{0}}^{T}\left(\|Y(t)-a(t)\|^{2}+\|u(t)\|^{2}\right) \mathrm{d} t \\
\text { s.t. } \\
\frac{\mathrm{d} Y(t)}{\mathrm{d} t}=A(t) Y(t)+B(t) u(t), t \in\left[t_{0}, T\right] \\
Y(T)=Y_{i}(T)_{i=1, \cdots, Q} \\
Y\left(t_{0}\right)=Y_{0}
\end{array}\right.
$$

$a_{i}(T)$ is defined and is a square integrable function on $\left[t_{0}, T\right]$ and such that

$$
\left\{\begin{array}{l}
a_{i}(t) \geq \frac{t}{T} \sqrt{\frac{1+N Z^{2}}{Q}}, i \in\{1, \cdots, Q\}, t \in\left[t_{0}, T\right] \\
\left\|\sum_{i=1}^{Q}\left(a_{i}(T)\right)^{2}-N Z^{2}\right\| \geq 1
\end{array}\right.
$$

where $Y_{\mathrm{i}}(T) \geq Z, \quad \forall i \in\{1, \cdots, Q\}, \quad u(t) \in \mathbb{R}^{p}$ with $u_{j}$ a continuous step function $\forall j=1, \cdots, p$.

\section{Theorem 2}

Under the assumption of controllability of the above system, the problem of minimization admits an optimal solution. Paths and optimal controls are obtained by resolving the following system,

$$
\left\{\begin{array}{l}
\frac{\mathrm{d} Y}{\mathrm{~d} t}=A(t) Y(t)-\frac{1}{2} B(t) B(t)^{t} \lambda(t) \\
\frac{\mathrm{d} \lambda}{\mathrm{d} t}=-(Y(t)-a(t))-A(t)^{t} \lambda(t) \\
Y\left(t_{0}\right)=Y_{0} \\
Y(T)=K, \text { where } Y_{i}=Z \\
\lambda(T) \perp T^{f}(Y(T))
\end{array}\right.
$$

where $\lambda(t)$ is the vector of Pontryagin multipliers and $T^{f}(Y(T))$ the space containing conditions on the Pontryagin multipliers. $T^{f}(Y(T))$ is defined in the Appendix.

Proof: (See the Appendix).

\section{A Parametric Illustration}

As an illustration we consider a general problem faces by a Government in determining dynamic poverty measures over time. To get a more interesting case, we consider a parametric problem.

\subsection{The Problem}

Consider a parametric dynamic optimization problem where government authorities have some flexibility on intermediary objectives as well as the control variables. The parametric dynamic optimization problem can be formalized as follows,

$$
\left\{\begin{array}{l}
\min J=\int_{0}^{T}\left(\left\|Y(t)-m_{1} a(t)\right\|^{2}+m_{2}\|u(t)\|^{2}\right) \mathrm{d} t \\
\text { s.t. } \\
\frac{\mathrm{d} Y}{\mathrm{~d} t}=A(t) Y(t)+B(t) u(t) \\
a_{i}(t) \geq \frac{t}{T} \sqrt{\frac{1+N Z^{2}}{Q}}, i=1, \cdots, Q \\
Y\left(t_{0}\right)=Y_{0} \\
Y(T)=\left(Y_{i}(T)\right), i=1, \cdots, Q
\end{array}\right.
$$


where $a_{i}(t)$ a functional such that $\int_{0}^{T}\left|a_{i}(t)\right|^{2} \mathrm{~d} t<+\infty$, $A \in M_{Q, Q}(\mathbb{R})$ and $B \in M_{Q, p}(\mathbb{R})$ and where for simplicity we assume that the coefficients of $A$ and $B$ are not time dependent. For the sake of flexibility, the objectives as well as the control variables are parameterized so as to account for possible changes during the implementtation of Government economic policies; $m_{1}, m_{2} \in \mathbb{R}^{+}$.

\subsection{Solution}

Using the Pontryagin principle, we get the following optimality system,

$$
\left\{\begin{array}{l}
\text { Min } \mathrm{J}: J=\int_{0}^{T}\left(\left\|Y(t)-m_{1} a(t)\right\|^{2}+m_{2}\|u(t)\|^{2}\right) \mathrm{d} t \\
\text { s.t. } \\
\frac{\mathrm{d} Y}{\mathrm{~d} t}=A Y(t)-\frac{1}{2 m_{2}} B B^{t} u(t) \\
\frac{\mathrm{d} \lambda}{\mathrm{d} t}=-2 Y(t)-A^{t} \lambda(t)+2 m_{1} a(t) \\
Y\left(t_{0}\right)=Y_{0} \\
Y(T)=\left(Y_{i}(T)\right), i=1, \cdots, Q \\
Y_{i}(T)=Z \\
\lambda(T) \perp T^{f}(Y(T))
\end{array}\right.
$$

Set $X=\left(\begin{array}{l}Y \\ \lambda\end{array}\right)$. We get,

$$
\left\{\begin{array}{l}
\frac{\mathrm{d} X}{\mathrm{~d} t}=\left(\begin{array}{ll}
A & -\frac{1}{2 m_{2}} B B^{t} \\
-2 I_{Q} & -A^{t}
\end{array}\right) X+\left(\begin{array}{l}
0 \\
2 m_{1} a(t)
\end{array}\right) \\
Y\left(t_{0}\right)=Y_{0} \\
Y_{i}(T)=Z, \quad i=1, \cdots, Q
\end{array}\right.
$$

The above differential system can be written as,

$$
\frac{\mathrm{d} X}{\mathrm{~d} t}=\underbrace{\left(\begin{array}{cc}
A & -\frac{1}{2 m_{2}} B B^{t} \\
-2 I_{Q} & -A^{t}
\end{array}\right)}_{C m_{2}} X+\underbrace{\left(\begin{array}{c}
0 \\
2 m_{1} a(t)
\end{array}\right)}_{F_{m_{1}}(t)}
$$

Or simply as, $\frac{\mathrm{d} X}{\mathrm{~d} t}=C m_{2} X+F_{m_{1}}(t)$

where $C_{m_{2}} \in M_{2 Q, 2 Q}(\mathbb{R})$ and $F_{m_{1}(t)} \in M_{2 Q, 1}(\mathbb{R})$.

Two cases must be considered depending on the fact that $C_{m_{2}}$ is diagonalizable or not. To conserve space, we consider only the first case and when eigenvalues are real and distinct. Note that, the optimality system even though linear, is too general in its expression and in the sign of the second member. The resulting general results will then be difficult to interpret. Let us consider a simple case where $Q=1$ and $p=1$. Again, for simplicity we assume that $A=(1)$ and $B=(1)$. The optimality system is therefore,

$$
\left\{\begin{array}{l}
\frac{\mathrm{d} Y}{\mathrm{~d} t}=Y(t)-\frac{1}{2 m_{2}} \lambda(t), m_{2}>0 \\
\frac{\mathrm{d} \lambda}{\mathrm{d} t}=-2 Y(t)-\lambda(t)+2 m_{1} a_{1}(t) \\
Y\left(t_{0}\right)=0 \\
\lambda(T) \perp T^{f}(Y(T))
\end{array}\right.
$$

To fix ideas consider that $a_{1}(t)=\frac{t}{T} \sqrt{\frac{1+N Z^{2}}{Q}}$. The underlying matrices are

$$
C_{m_{2}}=\left(\begin{array}{cc}
2 & -\frac{1}{2 m_{2}} \\
-2 & -1
\end{array}\right) \text { and } F_{m_{1}}(t)=\left(\begin{array}{c}
0 \\
\frac{2 m_{1} t}{T} \sqrt{\frac{1+N Z^{2}}{Q}}
\end{array}\right) .
$$

The eigenvalues of $C_{m_{2}}$ are

$\varphi_{1}=\sqrt{1+\frac{1}{m_{2}}}$ and $\varphi_{2}=-\sqrt{1+\frac{1}{m_{2}}}$ with $m_{2}>0 . C_{m_{2}}$ is diagonalizable. The matrix of associated eigenvectors $P$ is defined as,

$$
\begin{gathered}
P=\left(2 m_{2}\left(1-\sqrt{1+\frac{1}{m_{2}}}\right) 2 m_{2}\left(1+\sqrt{1+\frac{1}{m_{2}}}\right)\right) \\
P^{-1}=\left(\begin{array}{cc}
\frac{1+\sqrt{1+\frac{1}{m_{2}}}}{2 \sqrt{1+\frac{1}{m_{2}}}} & -\frac{1}{4 m_{2} \sqrt{1+\frac{1}{m_{2}}}} \\
1-\frac{1+\sqrt{1+\frac{1}{m_{2}}}}{2 \sqrt{1+\frac{1}{m_{2}}}} & \frac{1}{4 m_{2} \sqrt{1+\frac{1}{m_{2}}}}
\end{array}\right)
\end{gathered}
$$

and

The differential system is now equivalent to,

$$
\left(\begin{array}{c}
\frac{\mathrm{d} u_{1}}{\mathrm{~d} t} \\
\frac{\mathrm{d} u_{2}}{\mathrm{~d} t}
\end{array}\right)=\left(\begin{array}{cc}
\varphi_{1} & 0 \\
0 & \varphi_{2}
\end{array}\right)\left(\begin{array}{l}
u_{1} \\
u_{2}
\end{array}\right)+P^{-1} F_{m_{1}}(t)
$$

Solving, we get

$$
u_{1}(t)=c_{1} \mathrm{e}^{\varphi_{1} t}+\frac{m_{1}}{2 \varphi_{1} T m_{2}} \sqrt{\frac{1+N Z^{2}}{1+\frac{1}{m_{2}}}}\left(t+\frac{1}{\varphi_{1}}\right),
$$

where $c_{1}$ is a constant of integration. and 


$$
u_{2}(t)=c_{2} \mathrm{e}^{\varphi_{2} t}-\frac{m_{1}}{2 \varphi_{2} T m_{2}} \sqrt{\frac{1+N Z^{2}}{1+\frac{1}{m_{2}}}}\left(t+\frac{1}{\varphi_{2}}\right)
$$

$c_{2}$ being a constant of integration as well. Since, $U=P^{-1} X \Rightarrow X=P U$ where $X=\left(\begin{array}{c}Y(t) \\ \lambda(t)\end{array}\right)$, we obtain, $\left(\begin{array}{l}Y(t) \\ \lambda(t)\end{array}\right)=\left(2 m_{2}\left(1-\sqrt{1+\frac{1}{m_{2}}}\right) \quad 2 m_{2}\left(1+\sqrt{1+\frac{1}{m_{2}}}\right)\right)\left(\begin{array}{l}u_{1} \\ u_{2}\end{array}\right)$

After a bit of algebra we get,

$$
\left\{\begin{array}{l}
Y(t)=\left(c_{1} \mathrm{e}^{\varphi_{1} t}+c_{2} \mathrm{e}^{-\varphi_{1} t}\right)+\frac{m_{1} t}{\varphi_{1}^{2} T m_{2}} \sqrt{1+N Z^{2}} \\
\lambda(t)=2\left[\frac{-c_{1} \mathrm{e}^{\varphi_{1} t}}{\varphi_{1}+1}+\frac{c_{2} \mathrm{e}^{-\varphi_{1} t}}{\varphi_{1}-1}\right]+\frac{2 m_{1}(t-1) \sqrt{1+N Z^{2}}}{T \varphi_{1}^{2}}
\end{array}\right.
$$

The optimal control $u(t)$ is given by $u(t)=-\frac{1}{2 m_{2}} \lambda(t)$.

We now discuss several cases.

\subsection{Discussion}

Case 1: $m_{1} \rightarrow 0^{+}$and $m_{2} \rightarrow 0^{+}$.

$$
\begin{gathered}
Y(t) \rightarrow\left\{\begin{array}{l}
+\infty \text { if } c_{1}>0 \\
-\infty \text { if } c_{1}<0
\end{array}, \quad \lambda(t) \rightarrow\left\{\begin{array}{l}
+\infty \text { if } c_{1}<0 \\
-\infty \text { if } c_{1}>0
\end{array}\right.\right. \\
u(t) \rightarrow\left\{\begin{array}{l}
+\infty \text { if } c_{1}<0 \\
-\infty \text { if } c_{1}>0
\end{array}\right.
\end{gathered}
$$

and

Poverty can be greatly improved on the condition that intermediary objectives and control variables be realistic and comprehensive.

Case 2: $m_{1} \rightarrow 0^{+}$and $m_{2}$ fixed.

$$
Y(t) \rightarrow c_{1} \mathrm{e}^{\varphi_{1} t}+c_{2} \mathrm{e}^{-\varphi_{1} t}, \lambda(t) \rightarrow 2\left[\frac{-c_{1} \mathrm{e}^{\varphi_{1} t}}{\varphi_{1}+1}+\frac{c_{2} \mathrm{e}^{-\varphi_{1} t}}{\varphi_{1}-1}\right]
$$

and

$$
u(t) \rightarrow \frac{1}{m_{2}}\left[\frac{c_{1} \mathrm{e}^{\varphi_{1} t}}{\varphi_{1}+1}-\frac{c_{2} \mathrm{e}^{-\varphi_{1} t}}{\varphi_{1}-1}\right]
$$

Poverty can be gradually improved if intermediary objectives are reachable and control variables reasonably selected.

Case 3: $m_{1} \rightarrow 0^{+}$and $m_{2} \rightarrow+\infty$.

$$
Y(t) \rightarrow c_{1} \mathrm{e}^{t}+c_{2} \mathrm{e}^{-t}, \lambda(t) \rightarrow\left\{\begin{array}{l}
+\infty \text { if } c_{2}>0 \\
-\infty \text { if } c_{2}<0
\end{array}\right.
$$

and $u(t) \rightarrow-2 c_{2} \mathrm{e}^{-t}$

Poverty can be gradually improved but many control variables can create entropy in the system.
Case 4: $m_{1}$ fixed and $m_{2} \rightarrow 0^{+}$.

$$
Y(t) \rightarrow\left\{\begin{array}{l}
+\infty \text { if } c_{1}>0 \\
-\infty \text { if } c_{1}<0
\end{array}, \quad \lambda(t) \rightarrow\left\{\begin{array}{l}
+\infty \text { if } c_{1}<0 \\
-\infty \text { if } c_{1}>0
\end{array}\right.\right.
$$

and

$$
u(t) \rightarrow\left\{\begin{array}{l}
+\infty \text { if } c_{1}<0 \\
-\infty \text { if } c_{1}>0
\end{array}\right.
$$

Realistic intermediary objectives and well chosen control variables may result in positive impacts in terms of poverty alleviation.

Case 5: $m_{1}$ fixed and $m_{2}$ fixed.

The behaviors of $Y(t), \lambda(t)$ and $u(t)$ depend on the specific values assigned to $m_{1}$ and $m_{2}$.

Case 6: $m_{1}$ fixed and $m_{2} \rightarrow+\infty$.

$$
\begin{gathered}
Y(t) \rightarrow c_{1} \mathrm{e}^{t}+c_{2} \mathrm{e}^{-t}, \lambda(t) \rightarrow\left\{\begin{array}{l}
+\infty \text { if } c_{2}>0 \\
-\infty \text { if } c_{2}<0
\end{array}\right. \\
u(t) \rightarrow\left\{\begin{array}{l}
+\infty \text { if } c_{1}>0 \\
-\infty \text { if } c_{1}<0
\end{array}\right.
\end{gathered}
$$

Fixed objectives can be beneficiary for poverty improvement but many control variables can negatively affect the system.

Case 7: $m_{1} \rightarrow+\infty$ and $m_{2} \rightarrow 0^{+}$.

$Y(t), \lambda(t)$ and $u(t)$ oscillate to $\pm \infty$, a case of uncertainty.

Case 8: $m_{1} \rightarrow+\infty$ and $m_{2}$ fixed.

$Y(t) \rightarrow+\infty, \lambda(t) \rightarrow+\infty$ and $u(t) \rightarrow-\infty$

Many objectives with reasonable control variables may result in poverty improvement.

Case 9: $m_{1} \rightarrow+\infty$ and $m_{2} \rightarrow+\infty$.

$Y(t), \lambda(t)$ and $u(t)$ oscillate to $\pm \infty$, another case of uncertainty.

\section{Final Remarks}

How important are dynamic optimization techniques for poverty analysis? In dynamic settings, the paths of incomes are essential and the paper provides methods accordingly. It remains to establish optimality and stability criteria for the characterization of the various paths in a future research.

\section{References}

[1] P. G. Ciarlet, “Introduction à l'analyse Numérique Matricielle et à l’optimisation,” Dunod, Paris, 2006.

[2] J. M. Dia and D. Popescu, "Commande Optimale, Conception Optimisée des Systèmes,” Diderot Arts et Sciences, Paris, 1996.

[3] J. S. Rustagi, “Optimization Techniques in Statistics,” AP Harcourt Brace and Company Publishers, San Diego, 1997.

[4] R. Mart, "Optimisation Intertemporelles: Application aux 
Modèles Macroéconomiques,” Economica, Paris 1997.
Convexity,” Springer-Verlag, New York, 1980.

\section{Appendix: Theorems and Proofs}

\section{Theorem 1}

Let $t \in\left[t_{0}, T\right], Y \in \mathbb{R}^{Q}$ and $u_{0}:\left[t_{0}, T\right] \rightarrow \mathbb{R}^{p}$ be a vector of functions which represents control variables and $Y_{0}(\tau)$ the solution to,

$$
\left\{\begin{array}{l}
\dot{Y}(\tau)=A(\tau) Y_{0}(\tau)+B(\tau) u_{0}(\tau) . \\
\dot{Y}\left(t_{0}\right)=Y_{0}, \quad \tau \in\left[t_{0}, T\right]
\end{array}\right.
$$

Let $Y^{*}(\tau)$ be the solution to,

$$
\left\{\begin{array}{l}
\dot{Y}^{*}(\tau)=A(\tau) Y^{*}(\tau)+B(\tau) u^{*}(\tau) \\
\dot{Y^{*}}\left(t_{0}\right)=Y_{0}, \quad \tau \in\left[t_{0}, T\right]
\end{array}\right.
$$

where $u^{*}(\tau)=\Psi\left(\tau, Y^{*}(\tau)\right)$. The assumption on $\Psi$ insures the existence of the solution to the above system. To show that $\Psi$ is an optimal feedback control, we need to prove that,

$$
\begin{aligned}
& \int_{t_{0}}^{T}\left(\left\|Y^{*}(t)-a(t)\right\|^{2}+\left\|u^{*}(t)\right\|^{2}\right) \mathrm{d} t \\
& \leq \int_{t_{0}}^{T}\left(\left\|Y^{*}(t)-a(t)\right\|^{2}+\left\|u_{0}(t)\right\|^{2}\right) \mathrm{d} t
\end{aligned}
$$

Set, $f_{0}\left(t, Y^{*}(t), u^{*}(t)\right)=\left\|Y^{*}(t)-a(t)\right\|^{2}+\left\|u^{*}(t)\right\|^{2}$ and

$$
f_{0}\left(t, Y^{*}(t), u_{0}(t)\right)=\left(\left\|Y^{*}(t)-a(t)\right\|^{2}+\left\|u_{0}(t)\right\|^{2}\right)
$$

On the one hand,

$$
\begin{gathered}
V\left(T, Y^{*}(T)\right)-V\left(t_{0}, Y^{*}\left(t_{0}\right)\right)=\int_{t_{0}}^{T} \frac{\mathrm{d} V}{\mathrm{~d} t}\left(t, Y^{*}(t)\right) \mathrm{d} t \\
\frac{\mathrm{d} V}{\mathrm{~d} t}\left(t, Y^{*}(t)\right)=\frac{\partial V}{\partial t}\left(t, Y^{*}(t)\right)+\nabla_{Y} V\left(t, Y^{*}(t)\right) \frac{\mathrm{d} Y^{*}(t)}{\mathrm{d} t}
\end{gathered}
$$
and

$$
\frac{\mathrm{d} Y^{*}(t)}{\mathrm{d} t}=A(t) Y^{*}(t)+B(t) u^{*}(t)
$$

where $u^{*}(t)=\Psi\left(t, Y^{*}(t)\right)$. Since by assumption,

$$
\begin{aligned}
f_{0}\left(t, Y^{*}(t), D\right) \\
=\min \left\{f_{0}(t, Y(t), u(t))\right. \\
\left.\quad+\nabla_{Y} V(t, Y(t))[(A(t) Y(t)+B(t) u(t))]\right\}
\end{aligned}
$$

given $u \in \mathbb{R}^{p}$ where

$$
D=\Psi\left(t, Y^{*}(t)\right)+\nabla_{Y} V\left(t, Y^{*}(t)\right)
$$

We have,

$$
\times\left[A(t) Y^{*}(t)+B(t) \Psi\left(t, Y^{*}(t)\right)\right]
$$

$$
\begin{aligned}
0= & \frac{\partial V}{\partial t}+f_{0}\left(t, Y^{*}(t), \Psi\left(t, Y^{*}(t)\right)\right) \\
& +\nabla_{Y} V\left(t, Y^{*}(t)\right)\left[A(t) Y^{*}(t)+B(t) \Psi\left(t, Y^{*}(t)\right)\right] \\
= & \min \left\{f_{0}\left(t, Y^{*}(t), \Psi\left(t, Y^{*}(t)\right)\right)\right. \\
& \left.+\nabla_{Y} V\left(t, Y^{*}(t)\right)\left[A(t) Y^{*}(t)+B(t) \Psi\left(t, Y^{*}(t)\right)\right]\right\}
\end{aligned}
$$

given $u \in \mathbb{R}^{p}$

Thus,

$$
\begin{aligned}
& -f_{0}\left(t, Y^{*}(t), \Psi\left(t, Y^{*}(t)\right)\right) \\
& =\frac{\partial V}{\partial t}+f_{0}\left(t, Y^{*}(t), \Psi\left(t, Y^{*}(t)\right)\right) \\
& +\nabla_{Y} V\left(t, Y^{*}(t)\right)\left[A(t) Y^{*}(t)+B(t) \Psi\left(t, Y^{*}(t)\right)\right]
\end{aligned}
$$

Hence,

$$
\begin{aligned}
& V\left(T, Y^{*}(T)\right)-V\left(t_{0}, Y^{*}\left(t_{0}\right)\right) \\
& =-\int_{t_{0}}^{T} f_{0}\left(t, Y^{*}(t), \Psi\left(t, Y^{*}(t)\right)\right) \mathrm{d} t
\end{aligned}
$$

On the other hand,

$$
\begin{aligned}
& V\left(T, Y_{0}(T)\right)-V\left(t_{0}, Y_{0}\left(t_{0}\right)\right) \\
& =\int_{t_{0}}^{T} \frac{\mathrm{d} V}{\mathrm{~d} t}\left(t, Y_{0}(t)\right) \mathrm{d} t \\
& =\int_{t_{0}}^{T}\left[\frac{\partial V\left(t, Y_{0}(t)\right)}{\partial t}+\nabla_{Y} V\left(t, Y_{0}(t)\right) \frac{\mathrm{d} Y_{0}(t)}{\mathrm{d} t}\right] \mathrm{d} t
\end{aligned}
$$

We can immediately notice that $\forall u \in \mathbb{R}^{p}$, $f_{0}\left(t, Y_{0}(t), u(t)\right)+\nabla_{Y} V\left(t, Y_{0}(t)\right) \frac{\mathrm{d} Y_{0}(t)}{\mathrm{d} t}$ $\geq \min \left\{f_{0}(t, Y(t), u(t))+\nabla_{Y} V(t, Y(t)) \frac{\mathrm{d} Y(t)}{\mathrm{d} t}\right\}$ Hence,$$
=f_{0}\left(t, Y^{*}(t), \Psi\left(t, Y^{*}(t)\right)\right)
$$$$
+\nabla_{Y} V\left(t, Y^{*}(t)\right) \frac{\mathrm{d} Y^{*}(t)}{\mathrm{d} t} \text {, given } u \in \mathbb{R}^{p}
$$$$
V\left(T, Y_{0}(T)\right)-V\left(t_{0}, Y_{0}\left(t_{0}\right)\right)
$$$$
\geq-\int_{t_{0}}^{T} f_{0}\left(t, Y_{0}(t), u^{*}(t)\right) \mathrm{d} t
$$ 
We then conclude using the Hamilton-Jacobi-Bellman equation and the fact that $Y^{*}\left(t_{0}\right)=Y_{0}\left(t_{0}\right)=Y_{0}$, that

$$
\int_{t_{0}}^{T} f_{0}\left(t, Y^{*}(t), u^{*}(t)\right) \mathrm{d} t \leq \int_{t_{0}}^{T} f_{0}\left(t, Y_{0}(t), u_{0}(t)\right) \mathrm{d} t
$$

\section{Theorem 2}

We have,

$$
\left\{\begin{array}{l}
\min J: J=\int_{t_{0}}^{T}\left(\|Y(t)-a(t)\|^{2}+\|u(t)\|^{2}\right) \mathrm{d} t \\
\text { s.t. } \\
\frac{\mathrm{d} Y(t)}{\mathrm{d} t}=A(t) Y(t)+B(t) u(t), 0 \leq t \leq T \\
Y(0)=Y_{0} \\
Y(t)=\left(Y_{i}(T)\right)_{1 \leq i \leq Q}, Y_{i}(T)=Z, u(t) \in \mathbb{R}^{p}
\end{array}\right.
$$

with $u(t)$ a continuous step function, $\forall j=1, \cdots, p$. The Hamiltonian is,

$$
\begin{aligned}
& H(t, Y(t), u(t), \lambda(t)) \\
= & p_{0}\|Y(t)-a(t)\|^{2}+p_{0}\|u(t)\|^{2} \\
+ & \lambda(t)^{t}[A(t) Y(t)+B(t) u(t)]
\end{aligned}
$$

where $\lambda(t)$ is the vector of Pontryagin multipliers. The first order conditions are,

$$
\begin{gathered}
\frac{\partial H(t, Y(t), u(t), \lambda(t))}{\partial Y_{i}}=-\frac{\mathrm{d} \lambda_{i}}{\mathrm{~d} t}, \forall i=1, \cdots, Q \\
\frac{\partial H(t, Y(t), u(t), \lambda(t))}{\partial \lambda_{i}}=\frac{\mathrm{d} Y_{i}}{\mathrm{~d} t}, \forall i=1, \cdots, Q
\end{gathered}
$$

Thus,

$$
\begin{aligned}
& \frac{\partial H(t, Y(t), u(t), \lambda(t))}{\partial Y_{i}} \\
= & 2 p_{0}\left(Y_{i}(t)-a_{i}(t)\right)+\frac{\partial}{\partial Y_{i}}\left[{ }^{t} \lambda(t) A(t) Y(t)\right] \\
= & 2 p_{0}\left(Y_{i}(t)-a_{i}(t)\right)+\sum_{j=1}^{Q} \lambda_{j} \alpha_{j i}
\end{aligned}
$$

where $\alpha_{i i_{1 \leq i, j \leq Q}}=A(t)$. Finally, we get

$\frac{\partial H(t, Y(t), u(t), \lambda(t))}{\partial Y_{i}}=-\frac{\mathrm{d} \lambda(t)}{\mathrm{d} t}$. We now have the following differential system,

$$
\frac{\mathrm{d} \lambda(t)}{\mathrm{d} t}=-2 p_{0}\left(Y_{i}(t)-a_{i}(t)\right)-\sum_{j=1}^{Q} \lambda_{j} \alpha_{i_{1 j_{1 \leq i}} \leq Q}
$$

and $\lambda \perp T^{f}(Y)=\left\{\xi / G^{f}(Y(t)) \xi=0\right\}$. In our case, $G \equiv I_{d}\left(G^{f}=Y(t)\right)$; where

$$
\begin{gathered}
T^{f}=\left\{\frac{x}{G^{f}}-b^{f}=0\right\}, \\
T^{f}(Y(t))=\left\{\frac{\xi}{G^{f}}(Y(t)) \xi=0\right\}, \\
G^{f}: \mathbb{R}^{Q} \rightarrow \mathbb{R}^{Q}, \\
T^{0}=\left\{\frac{x}{g^{0}(x)}-b^{0}=0\right\}, T^{0}\left(Y_{0}\right)=\left\{\frac{\xi}{G^{0}\left(Y_{0}\right) \xi}=0\right\} .
\end{gathered}
$$

At the optimum, we have

$$
\begin{aligned}
\frac{\partial H(., .)}{\partial u_{j}} & =2 p_{0} u_{j}+\lambda(t)^{t} \frac{\partial}{\partial u_{j}}(B(t) u(t)) \\
& =0, \forall j \in\{1, \cdots, p\} \\
\Leftrightarrow & 2 p_{0} u_{j}(t)+B(t)^{t} \lambda(t)=0 .
\end{aligned}
$$

1) If $p_{0} \neq 0$, then $u(t)=-\frac{1}{2 p_{0}}\left(B(t)^{t} \lambda(t)\right)$.

2) If $p_{0}=0$, then $B(t)^{t} \lambda(t) \equiv 0$. By assumption we have, $\frac{\mathrm{d} Y(t)}{\mathrm{d} t}=A(t) Y(t)+B(t) u(t)$ which is controllable on $[0, T]$. Consider $\Phi(t, \tau)$ the function of transition matrices of $\frac{\mathrm{d} Y(t)}{\mathrm{d} t}=A(t) Y(t)$. The assumption of controllability is equivalent to

$$
\begin{aligned}
& \forall \xi \in \mathbb{R}^{Q}, \xi^{t} \Phi(t, \tau) B(\tau)=0, \\
& 0 \leq \tau \leq T \Rightarrow \xi=0
\end{aligned}
$$

We claim that if the system is controllable, then $p_{0} \neq 0$, otherwise from $\frac{\mathrm{d} \lambda}{\mathrm{d} t}=-2 p_{0}(Y(t)-a(t))-A(t)^{t} \lambda(t)$ we would have deduced, $\lambda(t)=\Phi^{t}(T, t) \lambda(T)$, and $B(t)^{t} \lambda(t)=0$. Thus, $\lambda(t)^{t} \Phi(T, t) B(t)=0,0 \leq t \leq T$. Hence, $\lambda(T)=0 \Rightarrow \lambda(t)=0$, which is one condition since $p_{0}$ and $\lambda(t)$ cannot both be equal to zero at a time. Therefore, $p_{0} \neq 0$. We can now normalize the system by setting $p_{0}=1$. The path and optimal control are obtained by solving the following system,

$$
\left\{\begin{array}{l}
\frac{\mathrm{d} Y(t)}{\mathrm{d} t}=A(t) Y(t)-\frac{1}{2} B(t)^{t} B(t) \lambda(t) \\
\frac{\mathrm{d} \lambda}{\mathrm{d} t}=-2(Y(t)-a(t))-A(t)^{t} \lambda(t) \\
Y(0)=Y_{0}, Y(t)=K=(Z, \cdots, Z)^{t} \\
Y_{i}(T)=Z, i=1, \cdots, Q
\end{array}\right.
$$

